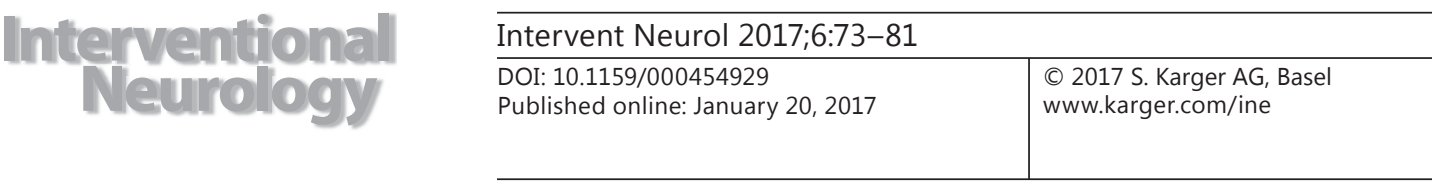

\title{
Embolization of Sacral Dural Arteriovenous Fistulas: A Case Series and Literature Review
}

\author{
Anthony M. Alvarado ${ }^{a}$ Diogo C. Haussen ${ }^{d}$ Koji Ebersole ${ }^{a}$ \\ Raul G. Nogueirad Michael G. Abraham ${ }^{\text {b, c }}$ \\ Departments of a Neurological Surgery, ${ }^{b}$ Neurology and ${ }^{c}$ Radiology, Kansas University \\ Medical Center, Kansas City, KS, and d Department of Neurology, Emory University/ \\ Grady Memorial Hospital - Marcus Stroke and Neuroscience Center, Atlanta, GA, USA
}

\section{Keywords}

Spinal dural arteriovenous fistula · Lateral sacral artery · Embolization · Superselective Wada testing

\begin{abstract}
The authors report 2 cases of sacral dural arteriovenous fistula (AVF) presenting with gradual neurologic decline characterized by progressive lower extremity weakness and bowel and bladder incontinence. Spinal angiography demonstrated a sacral dural AVF with perimedullary vein engorgement and drainage extending to the level of the cervical spine in both cases. The fistulas were completely obliterated with n-BCA ( $n$-butyl cyanoacrylate) embolic agent in one case, and with ethylene vinyl alcohol (Onyx) in the second, resulting in improvement of the symptoms in both patients. The unique features of this case report include the rare location of the fistula's origin, the necessity for complete spinal angiography, and the use of intraoperative monitoring in one case to guide embolization treatment.
\end{abstract}

(C) 2017 S. Karger AG, Basel

\section{Introduction}

Spinal dural arteriovenous fistulas (SDAVFs) are exceedingly rare; however, they compose an estimated $80 \%$ of spinal arteriovenous malformations [1-3]. Adding to the rarity of this entity, the origin of SDAVFs arising from the lateral sacral arteries is uncommon. While clinical manifestations and magnetic resonance imaging (MRI) may suggest SDAVF as the etiology for spinal cord dysfunction, a definitive diagnosis is difficult based on noninvasive 
Fig. 1. Sagittal T2-weighted magnetic resonance image showing diffuse cord hyperintensity and prominent intradural/extradural flow voids extending throughout the thoracolumbar spine.

\section{DOI: $10.1159 / 000454929$}

2017 S. Karger AG, Basel www.karger.com/ine

Alvarado et al.: Embolization of Sacral Dural Arteriovenous Fistulas: A Case Series and Literature Review

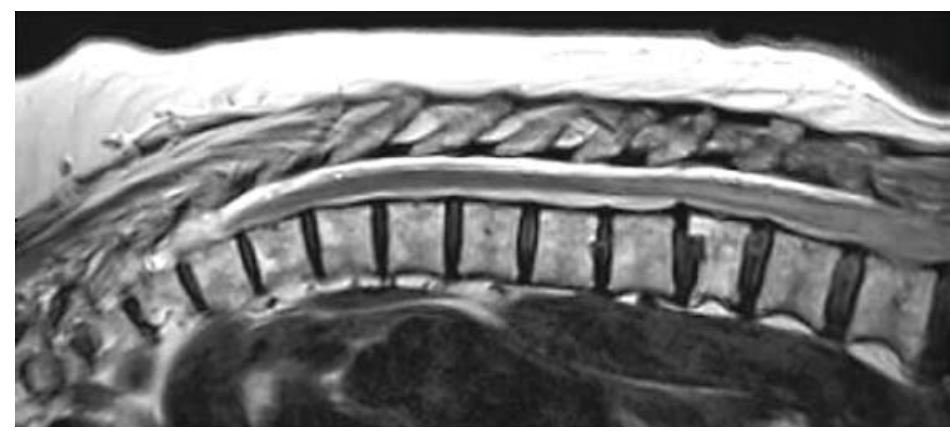

imaging; therefore, spinal angiography is necessary for a diagnosis. Without high clinical suspicion, misdiagnosis and delays in treatment may ensue. We report 2 cases of SDAVF that were successfully treated with endovascular embolization, one of which was performed using motor evoked potential monitoring and superselective Wada testing.

\section{Case 1}

History and Examination

A 78-year-old woman presented with a 6-month history of progressively worsening bilateral lower extremity weakness associated with diminished sensation. Two weeks prior she had begun experiencing lower back pain associated with a sharp burning pain radiating to the lower extremities. One week prior to presentation the pain had acutely worsened, resulting in spontaneous leg give-out and gait disturbance. Additionally, she had a several-year history of bowel and bladder incontinence following rectal surgery for a rectal fissure. Neurologic examination exhibited decreased sensation to light touch, pinprick, and proprioception in the bilateral lower extremities. Right lower extremity strength was $4 / 5$, with the remainder of the neurologic examination without abnormalities.

\section{Radiological Findings}

MRI with contrast of the thoracic and lumbar spine revealed a diffuse abnormal central-cord T2-weighted image hyperintensity extending from the T9-T10 level to the conus medullaris (Fig. 1). Additionally, numerous prominent intradural and extramedullary flow voids were visualized extending throughout the spinal cord along the posterior margin, most notably in the thoracolumbar region and terminating at the level of L4-L5. The findings were suggestive of an SDAVF. A complete diagnostic spinal angiogram, including both common iliac arteries, was performed. The angiogram demonstrated a venous pouch with early venous drainage into a perimedullary vein from the left sacral artery consistent with an SDAVF. The perimedullary vein coursed superiorly to the level of the cervical spinal cord and became tortuous and engorged at the level of the L1 vertebral body, which was seen throughout its course through the cervical spinal cord (Fig. 2, 3).

\section{Endovascular Treatment}

Following a thorough discussion of the potential risks associated with embolization of the vascular malformation, including permanent loss of sphincter function, the patient and family elected to proceed with endovascular embolization of the fistula given her history of bowel and bladder incontinence. The procedure was performed in the endovascular suite under general anesthesia. Given the risk of further neurologic deficit, endovascular treatment was performed using intraoperative neurophysiologic monitoring throughout the case. Following right femoral artery access, the left internal iliac artery was selectively catheterized with a 6-Fr/45-cm Arrow sheath (Teleflex, Morrisville, NC, USA). Under fluoroscopic and roadmap guidance, the left sacral artery was selected using an SL-10 microcatheter (Stryker Neurovascular, Fremont, CA, USA). Superselective angiography confirmed the left sacral AVF seen on the diagnostic angiogram.

Superselective Wada testing was performed with 3 aliquots of diluted lidocaine (30, 20, and $20 \mathrm{mg}$ ) into the left sacral artery, demonstrating loss of sphincter tone each time with return of function to baseline at 7 , 5 , and $4 \mathrm{~min}$, respectively, as seen on neurophysiologic monitoring. The injections of lidocaine were performed 
Fig. 2. a Left internal iliac artery injection demonstrating the left sacral artery with early venous drainage into a tortuous perimedullary vein extending to the lower thoracic level. b Magnification of the corresponding unsubtracted image, showing vascular malformation.

\begin{tabular}{l|l}
\hline DOI: $10.1159 / 000454929$ & $\begin{array}{l}\text { (c) 2017 S. Karger AG, Basel } \\
\text { www.karger.com/ine }\end{array}$ \\
\hline \multicolumn{1}{|l}{ Alvarado et al.: Embolization of Sacral Dural Arteriovenous Fistulas: A Case Series and }
\end{tabular}
Literature Review
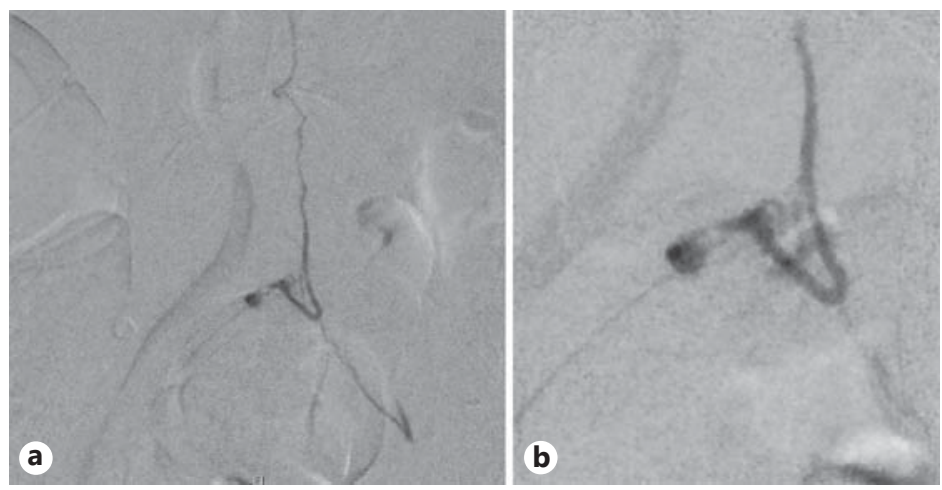

Fig. 3. Left internal iliac artery injection demonstrating early venous drainage into a tortuous perimedullary vein (arrows) extending to the cervical level (with the corresponding unsubtracted image).

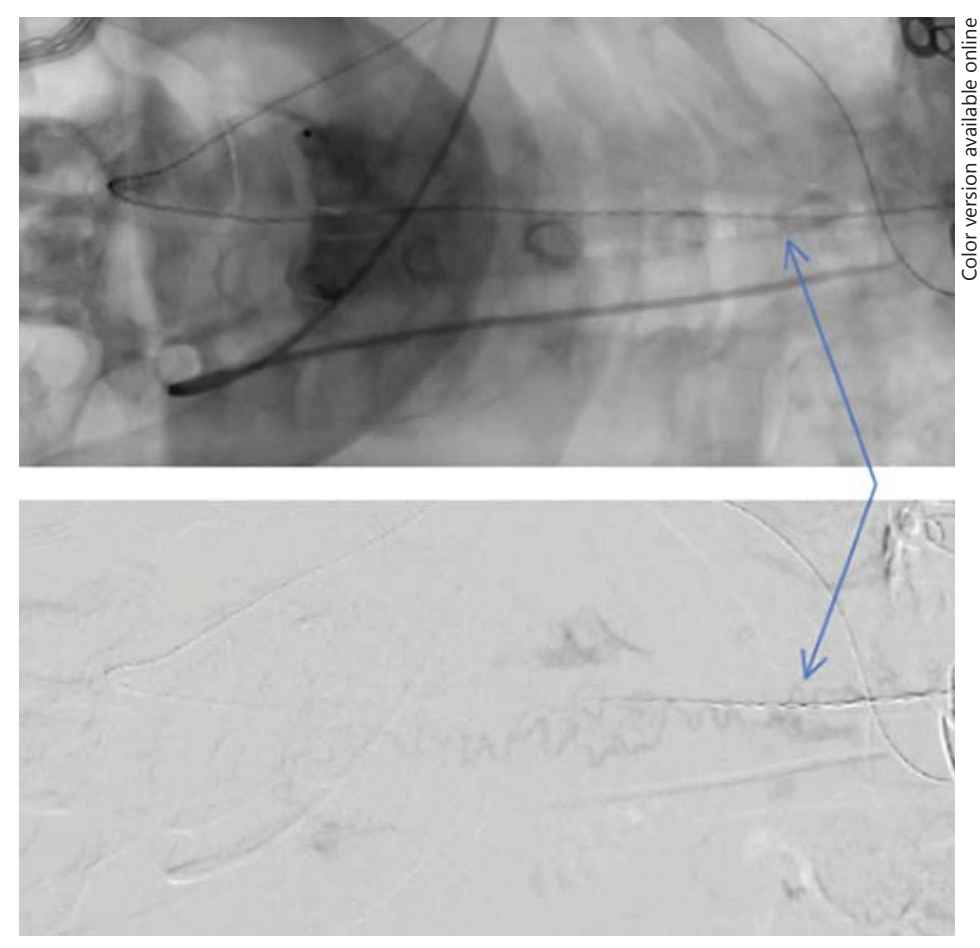

just proximal to the fistula, at the fistula, and in the right sacral artery just proximal to the fistula. The risks of permanent loss of sphincter tone were discussed a second time with the patient's family after Wada testing. Due to the already present bowel and bladder dysfunction, endovascular treatment was again decided on. New towels were placed over the working field, followed by infusion of three 3-mL syringes of dextrose. Embolization of the fistula was performed using $1 \mathrm{~mL}$ of $25 \% \mathrm{n}$-BCA ( $n$-butyl cyanoacrylate) glue (Cordis Neurovascular, Inc., Fremont, CA, USA) (Fig. 6). Following the infusion of n-BCA, sphincter motor responses remained intact, and did not terminate as had occurred with lidocaine administration. Postembolization angiography through the bilateral internal iliac arteries demonstrated complete obliteration of the AVF (Fig. 4, 5). There were no complications.

\section{Postoperative Course}

Following the intervention, the patient's neurologic examination remained stable without progression of neurologic dysfunction. The patient was discharged to inpatient rehabilitation and was discharged after 5 days without restrictions. At the 1-month follow-up, the patient demonstrated full strength, resolution of the bilateral lower extremity pain, and normal bladder function. Mild numbness and tingling in the lower extremities persisted. By the 3-month follow-up, motor strength and bladder function remained intact. 
Fig. 4. Post-stenting superselective left sacral artery angiogram.

\begin{tabular}{l|l}
\hline DOI: 10.1159/000454929 & $\begin{array}{l}\text { (c) 2017 S. Karger AG, Basel } \\
\text { www.karger.com/ine }\end{array}$ \\
\hline \multicolumn{1}{|l}{ Alvarado et al.: Embolization of Sacral Dural Arteriovenous Fistulas: A Case Series and }
\end{tabular}

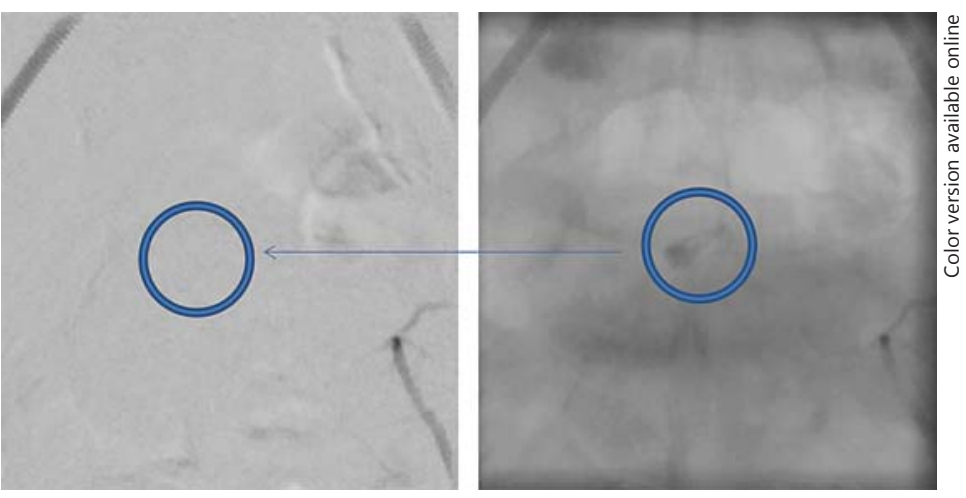

Fig. 5. Final left sacral artery angiogram demonstrating no further filling of the sacral dural AVF. The corresponding unsubtracted image demonstrates the n-BCA glue cast.

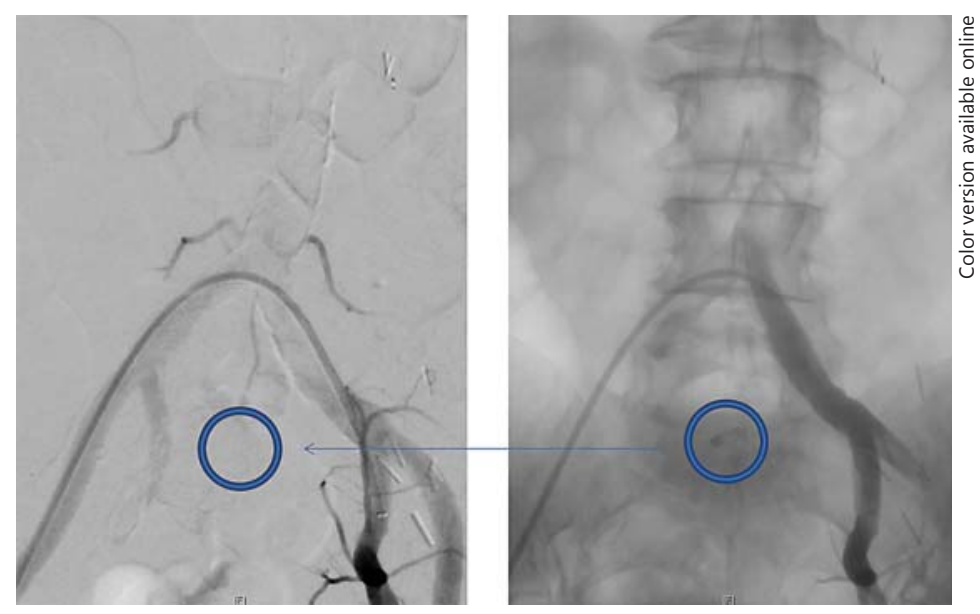

\section{Case 2}

\section{History and Examination}

A 69-year-old man with a history of coronary disease, lower extremity venous thrombosis after hip replacement, and long-standing lower back pain had experienced sudden bilateral leg cramping and numbness 2 years prior to presentation. Soon afterwards, bilateral lower extremity weakness had ensued and progressively worsened. Nine months prior to presentation, he had had difficulty ambulating and had started using a rolling walker. Four months prior to presentation, urinary retention and fecal incontinence had developed with progressive neurologic decline.

On initial presentation, his motor examination revealed slightly asymmetric strength findings: iliopsoas $3 / 5$, hamstrings and quadriceps $4-/ 5$, and dorsi-/plantar flexion $2 / 5$ in the more affected side. He could walk 10-15 feet at a time with a walker. All sensory modalities were impaired, and there was significant pain in the sacral area (9-10/10 in intensity) with exertion. Prior to presentation, he had received a course of steroids without significant clinical improvement following spinal axis imaging.

\section{Radiological Findings}

MRI of the thoracic and lumbar spine revealed a longitudinally extensive and expansive T2 hyperintense lesion from T7 to the conus medullaris (Fig. 7a). The lesion was slightly hypointense on T1-weighted imaging and had faint contrast enhancement. There was pial cord enhancement, mostly in the upper thoracic segments (Fig. 7b). T2-weighted imaging revealed possible flow voids in the perimedullary plexus (Fig. 7b, c). Angiography demonstrated a sacral dural AVF fed by the right lateral sacral artery and draining caudocranially via a radial vein into the perimedullary venous system (Fig. 8a). 
Alvarado et al.: Embolization of Sacral Dural Arteriovenous Fistulas: A Case Series and Literature Review
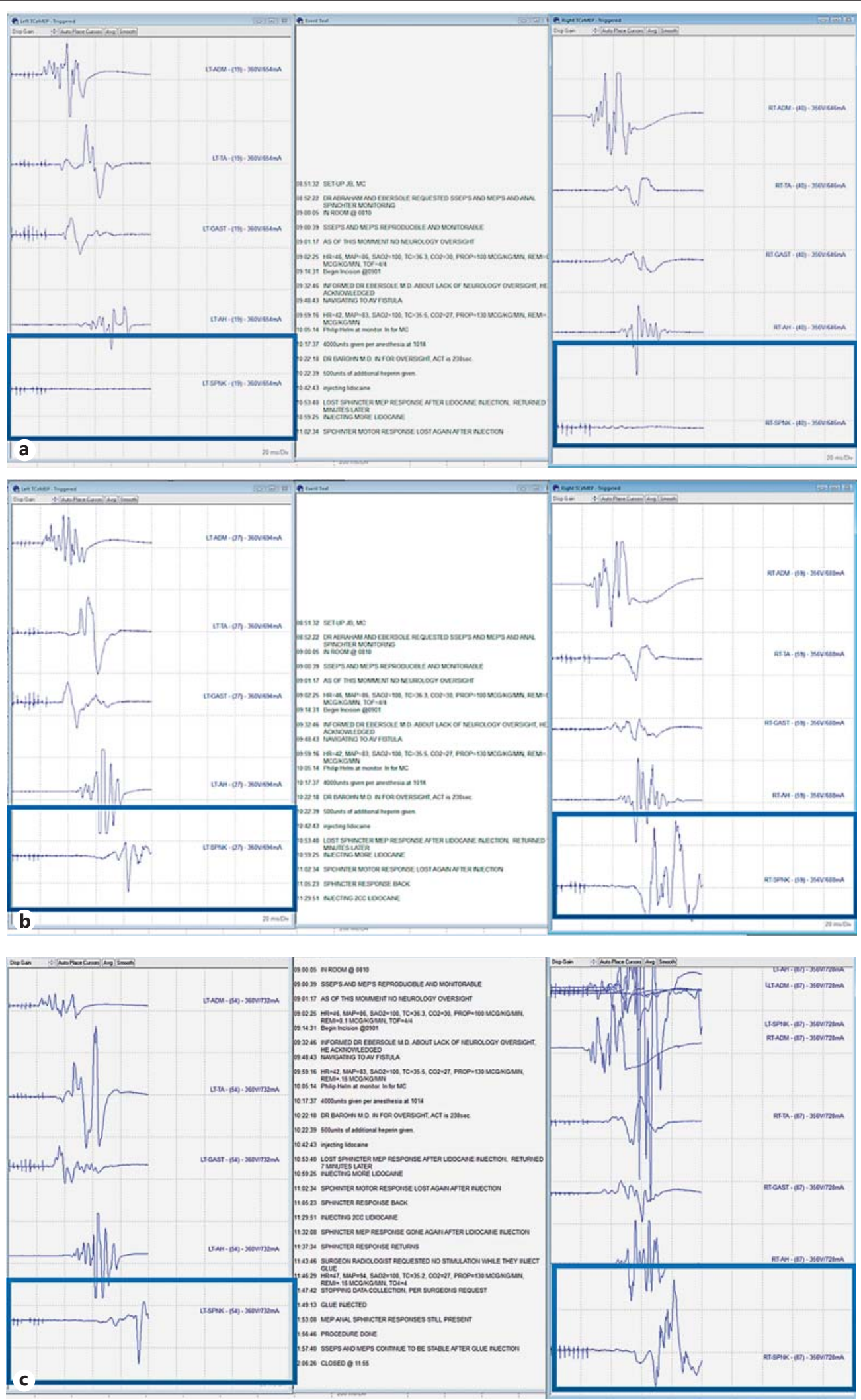

Fig. 6. a Intraoperative motor evoked potential after a 3-mL lidocaine injection. b Intraoperative motor evoked potential $12 \mathrm{~min}$ after response absence. $\mathbf{c}$ Intraoperative motor evoked potential after n-BCA injection. 
Alvarado et al.: Embolization of Sacral Dural Arteriovenous Fistulas: A Case Series and Literature Review

Fig. 7. a Sagittal thoracic T2weighted image. Arrow, extensive spinal lesion; arrowhead, flow voids. b Sagittal thoracic T1weighted image with gadolinium. Dashed arrow, pial enhancement. c Sagittal lumbar T2-weighted image. Arrowhead, flow voids.
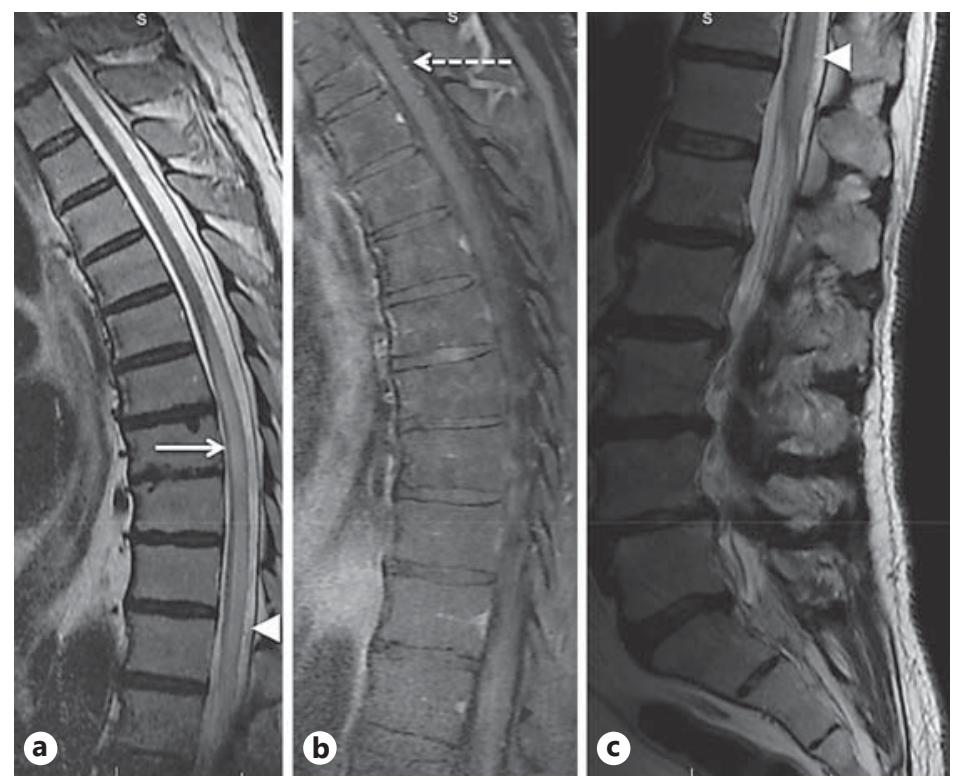

Endovascular Treatment

Through a 5-Fr Mikaelsson catheter (AngioDynamics, Queensbury, NY, USA), an Echelon-14 microcatheter (ev3 Inc., Plymouth, MN, USA) was navigated $4 \mathrm{~mm}$ from the fistulous point (Fig. 8a), and DMSO (dimethyl sulfoxide; $0.5 \mathrm{~mL}$ ) and ethylene vinyl alcohol (Onyx-18; ev3 Inc.; $0.8 \mathrm{~mL}$ ) were injected under negative roadmap guidance. The liquid embolic agent reached the venous system and provided reasonably dense opacification of the vein (Fig. 8b). The microcatheter was carefully retracted and a final angiogram showed very late contrast opacification of the draining vein, potentially indicating a residual small fistula (Fig. 8c).

\section{Postoperative Course}

Despite endurance and strength improvement, a follow-up angiogram performed 6 weeks after the embolization revealed residual fistula filling from a different lateral sacral artery radicular branch. A Marathon microcatheter (ev3 Inc.) was navigated and positioned $10 \mathrm{~mm}$ from the fistulous point (Fig. 8d, e). The inset in Figure 8e indicates the partial filling of the vein (related to previous laminar embolization and/ or further engorgement after recruitment of feeders). DMSO and Onyx-18 (1.5 mL) were injected until there was filling of the entire width of the draining vein with Onyx (Fig. 8f). Two months after the original embolization ( 2 weeks after re-embolization) there was improvement of motor function with 4 to $4+/ 5$ strength in all muscle groups. The patient was able to walk independently over 250 feet at a time, and the pain had improved to an intensity of 3/10. Urinary and bowel dysfunction were unchanged.

\section{Discussion}

SDAVFs are rare, acquired malformations that representan abnormal vascular connection between the arterial and venous systems. The connection is commonly between a branch of the radicular artery and a perimedullary vein [3]. The underlying pathophysiology in SDAVF is a venous hypertensive myelopathy, typically nonhemorrhagic, leading to impaired venous drainage, venous congestion, hypoxia, and potentially irreversible cord dysfunction [2, 3]. Despite the vast majority of SDAVF cases having an unclear origin, certain presentations provide an identifiable cause, including prior trauma or surgical intervention in the aforementioned area. Our patients reported histories of prior rectal surgery and hip replacement surgery, respectively, which escalated the clinical suspicion for SDAVF. Of the 42 cases Nishio et al. [4] reviewed, 4 cases were reported to have an inciting trauma etiology. 
Alvarado et al.: Embolization of Sacral Dural Arteriovenous Fistulas: A Case Series and Literature Review
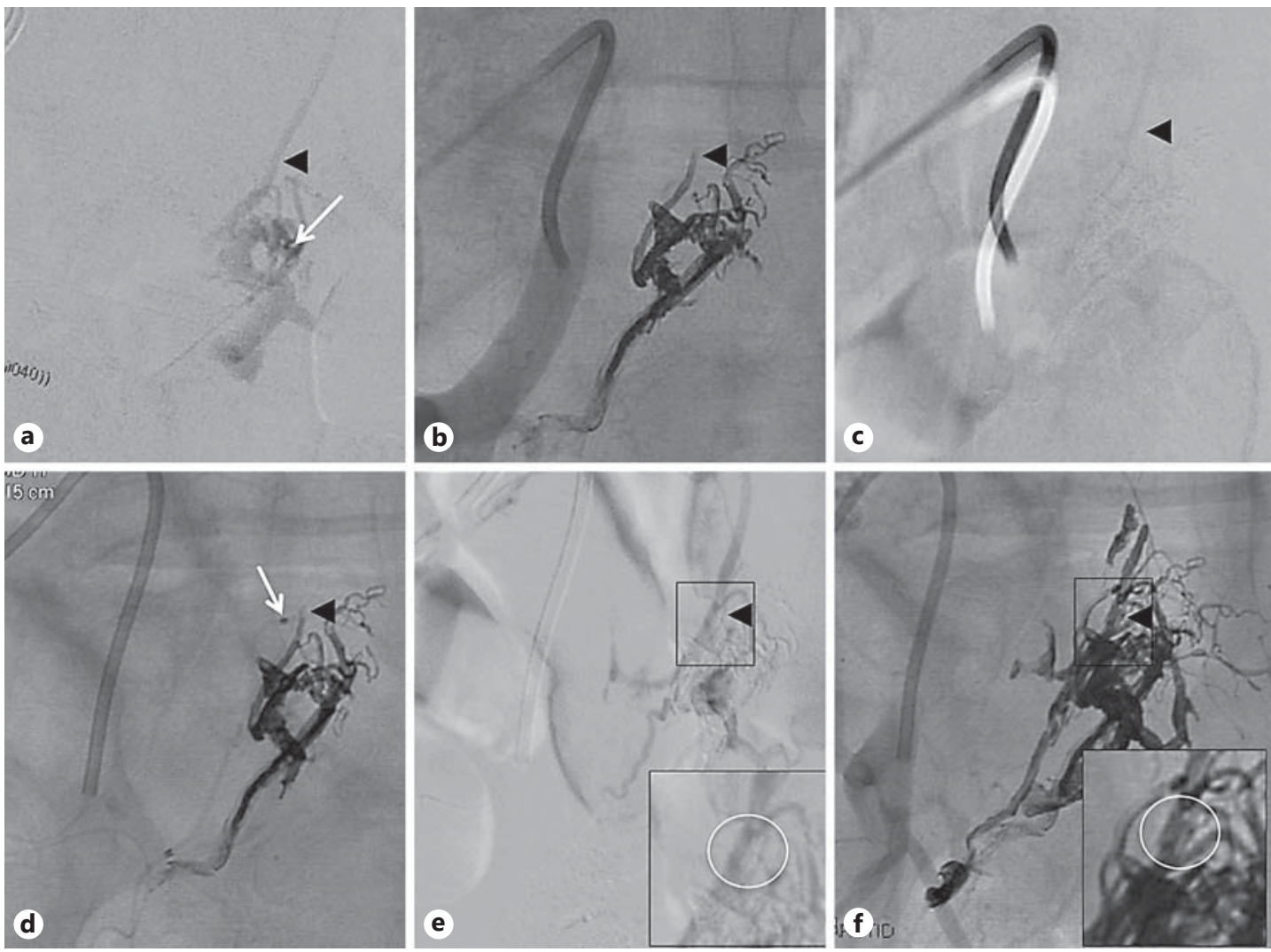

Fig. 8. a Subtracted microcatheter angiogram. Arrow, position of the microcatheter tip; arrowhead, draining vein. b Fluoroscopic single-shot X-ray image. Arrowhead, liquid embolic penetration in the draining vein. c Final subtracted right internal iliac angiogram in the venous phase. Arrowhead, late filling of the draining vein. d Fluoroscopic single-shot X-ray image. Arrow, position of the microcatheter tip on re-embolization; arrowhead, liquid embolic agent in the draining vein. e Subtracted microcatheter angiogram. Inset Draining partially obliterated by the previous procedure. Arrowhead, liquid embolic agent in the draining vein; circle, laminar penetration of the liquid embolic agent into the vein. $\mathbf{f}$ Final fluoroscopic single-shot X-ray image. Inset Complete obliteration of the vein by the liquid embolic agent (circle). Arrowhead, liquid embolic agent totally obliterating the draining vein.

Fistula sites often involved include the thoracic and lumbosacral region, with the segmental arteries supplying most fistulas [1-3, 5, 6]. Infrequently, they may arise in the sacral region, as in our cases. Larsen et al. [6] reported that the incidence of SDAVFs in the sacral region was $12.5 \%$ of all SDAVFs based on their series. In 2007, Nishio et al. [4] compiled 42 cases of SDAVFs arising from internal iliac arteries, including their case. However, some authors believe the actual incidence is much higher than reported in the literature, due to "negative" spinal angiograms and nonspecific clinical presentations of myelopathy attributed to other disorders [5, 6]. Nishio et al. [4] recommended that an angiographic examination of the internal iliac arteries be performed in patients with suspected SDAVF when the lesion has not been identified in the thoracic or lumbar region. Additionally, MRI findings are subtle and require an astute clinician to consider SDAVF in a differential diagnosis. Commonly reported MRI findings include an increased signal intensity on T2-weighted imaging, contrast enhancement, cord enlargement, and flow voids $[3,6]$. However, these subtle findings require a definitive diagnosis with a complete spinal angiography. Our patients had similar findings 
on their spinal MRI evaluation, including diffuse cord hyperintensity and numerous flow voids, consistent with the radiographic features of SDAVF.

Neurologic dysfunction in SDAVF is broad and demonstrates a high level of variability in regard to neurologic signs, symptoms, and disease progression. Most patients show some degree of dysfunction as demonstrated by progressive paraparesis, lower extremity sensory abnormality, and bowel and bladder dysfunction $[1-3,5,6]$. Despite the variable presentation of myelopathy and neurologic dysfunction in SDAVF, our patients reported progressive bilateral lower extremity weakness, pain, gait disturbance, and bowel and bladder incontinence, similar to cited cases of SDAVF $[2,3,6]$. Although our patients demonstrated improvement and/or resolution of pre-embolization symptoms and neurologic dysfunction, the clinical outcome and prognosis for neurologic improvement are highly variable, with longstanding cases tending to not necessarily improve much.

Following a definitive diagnosis of SDAVF, it is imperative to initiate therapy in a timely manner to prevent further neurologic insult and irreversible injury. Understanding the potential risk of permanent neurologic deficits following embolization of the SDAVF, mainly due to ischemic sequelae due to inadvertent embolization, a thorough conversation was held with both patients and their families prior to the intervention. Case 1 had selective angiography of the internal iliac arteries and underwent neurophysiologic monitoring (motor evoked potentials) and superselective Wada testing. These ancillary tests allow for continuous motor observation when intra-arterial infusion of a pharmacologic agent disrupts neural conduction to eloquent tissue, and therefore assists in predicting the safety of arterial embolization of the SDAVF prior to glue embolization. We chose lidocaine as it blocks sodium-gated channels, which are found in both gray and white matter [4].

When selecting an embolization material, Larsen et al. [6] reported that liquid embolization with cyanoacrylate glue was as effective as surgical obliteration in the treatment of SDAVFs in their study population. This is in contrast to using polyvinyl alcohol particle embolization, which has been associated with recanalization on follow-up angiography [3, 5]. For our patient population, n-BCA was utilized in case 1 and Onyx liquid embolic agent was utilized in case 2 . Both embolization agents were effective in fistula obliteration in our series, and overall have demonstrated relatively high cure rates compared to particle embolization in the existing literature.

\section{Conclusion}

We reported 2 rare cases of SDAVF supplied by the lateral sacral artery in the setting of prior surgeries. A history of previous surgeries and trauma may be shown to increase suspicion for the diagnosis. Awareness of this potential location is imperative, as incomplete spinal angiography will not elucidate an SDAVF arising from the lateral sacral arteries and will lead to deleterious delays in diagnosis. Further, the use of pre-embolization Wada testing can be beneficial in preserving motor function and preventing further neurologic decline in the setting of endovascular therapy of SDAVFs. In conclusion, endovascular therapy was a feasible, effective, and safe approach to treatment of SDAVF in our patients. Neurophysiologic monitoring and superselective Wada testing may optimize the treatment course.

\section{Disclosure Statement}

The authors have no financial disclosures or affiliations to acknowledge. 
Alvarado et al.: Embolization of Sacral Dural Arteriovenous Fistulas: A Case Series and Literature Review

\section{References}

1 Schaat TJ, Salzman KL, Stevens EA: Sacral origin of a spinal dural arteriovenous fistula: case report and review. Spine (Phila Pa 1976) 2002;27:893-897.

2 O'Brien WT, Schwartz ED, Hurst RW, Sinson G: Spinal dural arteriovenous fistula with supply from sacral arteries. Surg Neurol 2001;56:175-176; discussion 177.

3 Fitzsimmons BF, Marshall RS, Pile-Spellman J, Lazar RM: Neurobehavioral differences in superselective Wada testing with amobarbital versus lidocaine. AJNR Am J Neuroradiol 2003;24:1456-1460.

4 Nishio A, Ohata K, Takami T, Nishikawa M, Hara M: Atypical spinal dural arteriovenous fistula with supply from the lateral sacral artery. J Clin Neurosci 2007;14:65-68.

5 Park KW, Park SI, Im SB, Kim BT: Spinal dural arteriovenous fistula with supply from the lateral sacral artery - case report and review of literature. J Korean Neurosurg Soc 2009;45:115-117.

6 Larsen DW, Halbach VV, Teitelbaum GP, McDougall CG, Higashida RT, Dowd CF, Hieshima GB: Spinal dural arteriovenous fistulas supplied by branches of the internal iliac arteries. Surg Neurol 1995;43:35-40; discussion 40-41. 